\title{
Mobile-Assisted Cognitive Behavioral Therapy for Negative Symptoms: Open Single-Arm Trial With Schizophrenia Patients
}

Eric Granholm ${ }^{1}, \mathrm{PhD}$; Jason Holden ${ }^{2}, \mathrm{PhD}$; Kristen Dwyer ${ }^{2}, \mathrm{PhD}$; Tanya Mikhael $^{2}$, BA; Peter Link ${ }^{2}$, MA; Colin $\operatorname{Depp}^{1,2}, \mathrm{PhD}$

${ }^{1}$ VA San Diego Healthcare System, San Diego, CA, United States

${ }^{2}$ Department of Psychiatry, University of California, San Diego, San Diego, CA, United States

Corresponding Author:

Eric Granholm, PhD

VA San Diego Healthcare System

9500 Gilman Drive \#0737

San Diego, CA

United States

Phone: 18585342542

Email: egranholm@ucsd.edu

\section{Abstract}

Background: Negative symptoms are an important unmet treatment need for schizophrenia. This study is a preliminary, open, single-arm trial of a novel hybrid intervention called mobile-assisted cognitive behavioral therapy for negative symptoms (mCBTn).

Objective: The primary aim was to test whether mCBTn was feasible and could reduce severity of the target mechanism, defeatist performance attitudes, which are associated with experiential negative symptoms and poor functioning in schizophrenia.

Methods: Participants with schizophrenia or schizoaffective disorder $(\mathrm{N}=31)$ who met prospective criteria for persistent negative symptoms were enrolled. The blended intervention combines weekly in-person group therapy with a smartphone app called CBT2go. The app extended therapy group skills, including recovery goal setting, thought challenging, scheduling of pleasurable activities and social interactions, and pleasure-savoring interventions to modify defeatist attitudes and improve experiential negative symptoms.

Results: Retention was excellent ( $87 \%$ at 18 weeks), and severity of defeatist attitudes and experiential negative symptoms declined significantly in the mCBTn intervention with large effect sizes.

Conclusions: The findings suggest that $\mathrm{mCBT} n$ is a feasible and potentially effective treatment for experiential negative symptoms, if confirmed in a larger randomized controlled trial. The findings also provide support for the defeatist attitude model of experiential negative symptoms and suggest that blended technology-supported interventions such as mCBTn can strengthen and shorten intensive psychosocial interventions for schizophrenia.

Trial Registration: ClinicalTrials.gov NCT03179696; https://clinicaltrials.gov/ct2/show/NCT03179696

(JMIR Ment Health 2020;7(12):e24406) doi: $\underline{10.2196 / 24406}$

\section{KEYWORDS}

motivation; persistent negative symptoms; dysfunctional attitudes; mHealth; blended intervention; mobile phone

\section{Introduction}

Negative symptoms account for much of the poor functional outcome in schizophrenia and are an unmet treatment need [1-3]. Negative symptoms can refer to reduced expressive (eg, facial affect and voice tone) or experiential (eg, avolition and asociality) symptoms, which comprise two separate factors [4-6]. Experiential negative symptoms are particularly important to treat, because they are strongly associated with functioning
$[5,7]$. Unfortunately, available pharmacological treatments have only limited benefits for negative symptoms [8,9].

Beck and colleagues [10-12] have proposed that interventions that reduce defeatist attitudes may improve negative symptoms and functioning in schizophrenia. Several studies have found that cognitions such as defeatist performance (eg, "Why try, I always fail") and social disinterest (eg, "I'm better off alone") attitudes are associated with negative symptoms, and to some extent poor functioning, even after accounting for depression 
[10,13-18]. In social learning theory [19], self-competency beliefs are also central to motivation for achievement and engagement in effortful goal-directed activities. The Beck model hypothesis is that defeatist attitudes lead to low motivation and avoidance of effortful goal-directed activities. Thus, an intervention that targets defeatist attitudes may increase motivation and effort toward goal-directed activities.

Defeatist attitudes can be targeted in cognitive behavioral therapy (CBT). Clinical trials of CBT for psychosis have found mixed results for reducing negative symptoms [20,21], but some CBT interventions that specifically targeted defeatist attitudes have found more promising results [22-27]. Social skills training (SST) has also produced significant but modest improvements in negative symptoms [28,29]. In our cognitive behavioral social skills training (CBSST) intervention, which combines CBT and SST [30], we have found significant improvements in defeatist attitudes and in experiential negative symptoms and functioning; improvements in experiential negative symptoms were mediated by improvements in defeatist attitudes [23]. Modification of defeatist attitudes, therefore, may be a mechanism of change in CBSST, whereby reduction in defeatist attitudes contributes to increased motivation and effort toward goal-directed tasks. CBSST, however, is an intensive and lengthy (ie, up to 36 sessions), high-burden, multicomponent intervention. If the intervention could be shortened and the focus on reducing defeatist attitudes strengthened by using mobile interventions, the cost and burden of CBSST implementation could be reduced. In this way, blended interventions that combine mobile interventions with in-person interventions could increase access to evidence-based interventions for schizophrenia by reducing implementation barriers.

Smartphones are widely available, affordable, and frequently used by individuals with serious mental illness [31-34]. In previous clinical trials, we have found significant improvements in social functioning and symptoms in individuals with schizophrenia and bipolar disorder using 12-week mobile interventions that incorporate CBT principles with minimal therapist contact [35,36]. A number of other mobile interventions for schizophrenia have been developed and received preliminary testing, but few have specifically targeted negative symptoms or motivation and few have blended in-person plus app interventions; for reviews, see Camacho et al [37] and Firth and Torous [38]. One recent pilot trial of a blended intervention for psychosis [39] used a brief coping-focused intervention for distressing voices-SAVVy (Smartphone-Assisted coping-focused interVention for Voices)—which blended four sessions of in-person therapy with ecological momentary assessment (EMA) or ecological momentary intervention between sessions; the trial found that the intervention was feasible, improved coping, and, at a trend level, reduced severity of voices. Another app-only trial that did target motivation in schizophrenia-PRIME (Personalized Real-time Intervention for Motivational Enhancement) $[40,41]$-led to improvements in self-efficacy beliefs and social interactions with peers, as well as reduced defeatist beliefs and self-reported negative symptoms in individuals with recent-onset schizophrenia. These trials support the feasibility and potential benefits of this approach.
Given the promise of in-person and mobile CBT interventions targeting defeatist attitudes and motivation in schizophrenia, we developed a blended intervention, called mobile-assisted cognitive behavioral therapy for negative symptoms (mCBTn), which combines in-person, 90-minute, weekly groups with a mobile app called CBT2go. The mCBTn intervention primarily targets defeatist attitudes to improve experiential negative symptoms in schizophrenia. The CBT components and skills-training approach of our CBSST group intervention were combined with mobile thought-challenging interventions that were based on our Mobile Assessment and Treatment of Schizophrenia (MATS) [35] and CBT2go [36] interventions. This thought-challenging algorithm incorporated EMA to sample attitudes and moods in real-world contexts and then used personalized evidence to challenge dysfunctional beliefs (eg, if a participant rates their expectation for pleasure in a planned social interaction at a clubhouse as low, they would receive the challenge, "But you said you had fun at the clubhouse last time"). In addition to thought challenging, the CBT2go app and group intervention used in $\mathrm{mCBTn}$ both also incorporated recovery goal setting and tracking, scheduling pleasurable activities and social interactions, and pleasure-savoring interventions.

We conducted an open preliminary trial of mCBTn in patients with schizophrenia or schizoaffective disorder with moderate to severe persistent negative symptoms, and hypothesized that defeatist attitudes and experiential negative symptoms would be significantly reduced from baseline to end of treatment. This open trial was funded as part of the National Institute of Mental Health Experimental Therapeutics Program (RFA-MH-18-704 R61/R33), which involves preliminary testing of an intervention's impact on a target mechanism (ie, defeatist attitudes) associated with an important clinical outcome (ie, experiential negative symptoms). The primary aim of the study was target engagement; that is, we hypothesized that mCBTn would lead to a significant reduction in severity of defeatist attitudes. We also assessed participants at 12, 18, and 24 weeks of treatment to determine which dose of treatment could produce at least a medium effect size (Cohen $d=0.5$ ) improvement in defeatist attitudes. In the Experimental Therapeutics Program, contingent on changing the target in this single-arm open-trial phase, a larger randomized controlled trial (RCT) will be conducted with treatment at the dose identified.

\section{Methods}

This was a single-arm, open-trial, pre-post evaluation of the feasibility and preliminary effect of mCBTn. This trial was registered at ClinicalTrials.gov (NCT03179696).

\section{Sample}

The study protocol was reviewed and approved by the Institutional Review Board of the University of California, San Diego, prior to initiating research activities with participants. Participants with schizophrenia or schizoaffective disorder were selected who have moderate to severe persistent experiential negative symptoms [3], as well as moderate to severe defeatist attitudes, recognizing that an intervention targeting defeatist attitudes is not likely to be helpful for consumers who do not 
have them. Participants were required to meet all inclusion and exclusion criteria over a 2 -week evaluation phase. Inclusion criteria were as follows:

1. Voluntary informed consent to participate and capacity to consent.

2. Aged 18-65 years.

3. Diagnostic and Statistical Manual of Mental Disorders, fifth edition (DSM-5), diagnosis of schizophrenia or schizoaffective disorder based on a Structured Clinical Interview for DSM-5 (SCID-5) interview and available medical record review.

4. Moderate to severe experiential negative symptoms in at least two of the three Clinical Assessment Interview for Negative Symptoms [5] Motivation and Pleasure (CAINS-MAP) domains (mean score of 2 [ie, moderate] or greater for items averaged within the Social, Work, or Recreational domain) at the beginning and end of the 2-week evaluation phase.

5. Moderate to severe defeatist attitudes (score of $>50$ on the Defeatist Performance Attitude Scale [DPAS] [10]).

6. A 6th grade or higher reading level on the Wide Range Achievement Test-4 Reading subtest.

7. Clinically stable and on stable medications (ie, no hospitalizations or medication changes in 4 months prior to enrollment).

Exclusion criteria were as follows:

1. Prior CBT in the past 2 years.

2. Greater than moderate Positive and Negative Syndrome Scale (PANSS) [42] positive symptoms (score $>5$ for any item: item P1 [delusions], item P2 [disorganization], item P3 [hallucinations], or item P6 [suspiciousness]).

3. Severe depression on the Calgary Depression Scale for Schizophrenia (CDS) [43] (score >8).

4. Extrapyramidal symptoms: Simpson-Angus Scale [44] (score >7).

5. DSM-5 alcohol or substance use disorder in past 3 months based on the SCID-5.

6. Level of care required interferes with outpatient therapy (eg, hospitalized or severe medical illness).

7. Unable to adequately see or manually manipulate the mobile device.

\section{Blended mCBTn Intervention}

The therapy group and the mobile app integrated skills-based interventions, including recovery goal setting, thought challenging, scheduling of pleasurable activities and social interactions, and pleasure-savoring interventions to modify defeatist attitudes and improve motivation and pleasure negative symptoms. A modified 12-session version of the Cognitive Skills Module of CBSST [30] was delivered in 90-minute, weekly group therapy sessions with two masters-level therapists and approximately 6 participants per group. The 12-session module provided all the core mCBTn content within the minimum number of sessions that might be identified as the optimal dose, but the module was repeated for a total of 24 sessions to determine if more sessions were needed to change the defeatist attitude target.
The mCBTn manual included a therapist guide and a patient workbook describing the skills and homework assignments, as well as a collection of games and exercises to make learning fun and promote engagement [30]. The module began with setting a meaningful living, learning, working, or socializing recovery goal, and the goal was broken down into short-term goals and goal steps. Defeatist attitudes and avoidance behaviors that interfere with working on the goal were then modified using CBT skills. Group members were introduced to the general concepts of CBT, including the relationship between thoughts, actions, and feelings (ie, generic cognitive model); thought challenging through behavioral experiments and examining evidence for beliefs; and mistakes in thinking. The primary thought-challenging skill trained was the $3 \mathrm{Cs}$ : Catch It, Check It, Change It- "It" is an unhelpful defeatist thought. Group time was also spent practicing the smartphone interventions, developing content for the mobile device (eg, personalized motivational and thought-challenging statements about goals, socializing, and pleasurable activities), and reviewing data collected with the device to challenge defeatist attitudes.

An iPhone $5 \mathrm{~s}$ or $5 \mathrm{SE}$ was provided to all participants with an unlimited data plan and could receive and send unlimited texts and phone calls. Mobile interactions were triggered by an app notification in the morning, with reminder prompts at midday and evening. If participants responded to the notification earlier in the day, the second and/or third notifications were not delivered. The purpose of the notifications was to prompt daily engagement with the app. Device training was provided on how to operate and charge the device, the meaning of all questions and response choices, procedures for carrying the device, responding to prompts, how to access crisis lines, and how to use various apps. This information was also provided in a written manual given to participants. Participants returned the device at the end of treatment.

The CBT2go app was used to prompt and track each group member's goal-directed activities in the community, facilitate adherence to homework assignments involving community practice of thought-challenging skills trained in group, and prompt performance and savoring of personalized pleasurable activities and social interactions planned in group. The CBT2go app used personalized statements developed in group to challenge social disinterest and defeatist attitudes in real-time, real-world environments. After groups, therapists could enter personalized comments that participants made in group into a web-based dashboard (eg, "Having coffee with Jim is fun" and "Angie always makes you laugh"), which were used by the app to challenge low expectation ratings of motivation, anticipatory pleasure, or anticipated success for planned activities (see sample screenshots in Figure 1).

Participants carried the device and received the mobile intervention for the entire 24-week, blended group-plus-app intervention period. Each day during treatment, the CBT2go app alerted participants in the morning to make an action plan, which involved responding to a multiple-choice question about what to do that day: (1) a goal step, (2) social interaction, (3) pleasurable activity, or (4) homework assignment, or take the day off. Following the participant's choice, the CBT2go app asked for three EMA ratings (slide bar ranging from 1 [not at 
all] to 7 [very much]) of (1) motivation to do the activity, (2) anticipated success, and (3) anticipated pleasure for the action plan. High ratings were reinforced (eg, "That's right, socializing can be fun"), and low ratings were challenged (eg, "Don't forget, you said walking on the beach was fun" or "But your goal is to make a close friend"). Behavioral experiments were then suggested to test out beliefs (eg, "Try asking someone to go for a short walk"). If a pleasurable activity action plan was selected, the pleasurable activities component of the CBT2go app was opened, which displayed several personalized activities that the participant previously entered. For the selected activity, the time and place to do the activity was queried (ie, a reminder alert was delivered at the time planned) and anticipated pleasure ratings were queried on a slide bar ranging from 0 (low) to 10 (high). Pleasure was again rated if the activity was completed, and these ratings were saved and available on demand in the group sessions for the therapist to discuss how people often think activities will not be as fun as they turn out to be and how this low expectation can reduce the likelihood of doing the activity. The app also prompts pleasure savoring of completed activities by taking a selfie or other photo or journaling about their experiences in the app. These photos and journal entries are also available on demand for review by participants or therapists in group sessions. Therapists helped develop the plans for pleasurable activities in group. Finally, if homework was the action plan selected, participants were directed to use their homework sheets and workbooks from the group to complete their weekly homework assignment.

Finally, an on-demand recovery goal-setting component of the CBT2go app was also provided and was populated during the goal-setting sessions in group by therapists and participants, as well as between sessions for homework by participants. A long-term goal was set and short-term goals and goal steps that would facilitate achievement of the long-term goal were entered into the app. The app could be accessed on demand to remind participants of goals, and goal steps could be checked off to track and motivate goal progress.

Figure 1. Screenshots of the CBT2go app for planning pleasurable or social activity. Low motivation, high defeatist attitudes (ie, anticipated success), or low anticipatory pleasure ratings triggered personalized reappraisal evidence. A separate My Activities tool was used to plan and savor activities.

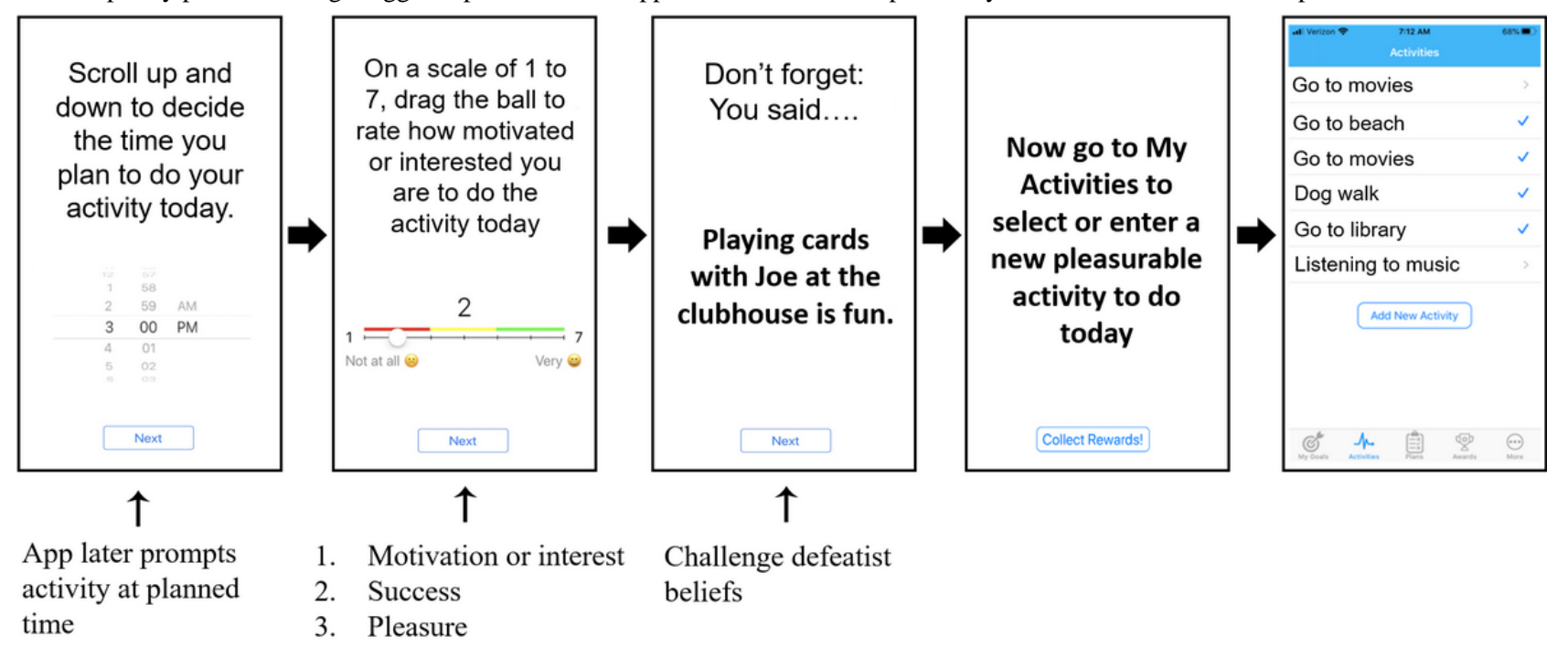

\section{Assessments}

Participants were assessed at 2 weeks prior to baseline; at baseline; and at 12, 18, and 24 weeks of treatment. Dysfunctional attitudes were measured on the DPAS [10] (ie, primary target mechanism) and the Asocial Beliefs Scale (ABS) [45]. The CAINS-MAP [5] subscale was the primary negative symptom outcome measure. The CDS [43] and the PANSS [42] positive symptom subscale were used to assess secondary symptom outcome domains. Functioning was assessed on the Abbreviated Quality of Life Scale (A-QLS) [46] and the Social Functioning Scale (SFS) [47].

\section{Statistical Analyses}

Mixed-effects regression models, utilizing HLM (hierarchical linear modeling) v6.08 (Scientific Software International), were estimated to predict each in-lab outcome assessment and mobile CBT2go app ratings of motivation, success, and anticipated pleasure for activities using time in weeks since baseline as a level-1 predictor. Paired-sample, 2-tailed $t$ tests between baseline and each follow-up assessment tested whether significant change was found for each outcome at each assessment point to inform the dose of treatment that might achieve a significant improvement in DPAS and CAINS-MAP scores with at least a medium effect size (Cohen $d=0.5$ ).

\section{Results}

\section{Sample}

We recruited and assessed 67 participants; see the CONSORT (Consolidated Standards of Reporting Trials) diagram in Figure 2. However, 36 of the 67 participants (54\%) were excluded because they did not meet persistent negative symptom criteria. For the 31 participants enrolled in treatment, excellent retention was found with $28(90 \%), 27(87 \%)$, and $25(81 \%)$ participants assessed at the 12-, 18-, and 24-week assessment points, respectively. There was only one adverse event, which was a hospitalization for symptom exacerbation in the context of medication nonadherence. The participant remained in the study. The sample had a mean age of 48.3 (SD 9.5) years and a mean of 11.8 (SD 1.5) years of education. The sample was $65 \%$ 
(20/31) male, was 65\% (20/31) White, and had moderate baseline symptom severity-the PANSS total mean score was 60.5 (SD 12.7). Out of 31 participants, $21(68 \%)$ had a schizophrenia diagnosis and $10(32 \%)$ had schizoaffective disorder. Table 1 shows descriptive statistics and comparisons between baseline and each assessment point for all outcome variables.

Figure 2. CONSORT (Consolidated Standards of Reporting Trials) flow diagram of participants through the open trial. CBT: cognitive behavioral therapy; DPAS: Defeatist Performance Attitude Scale.

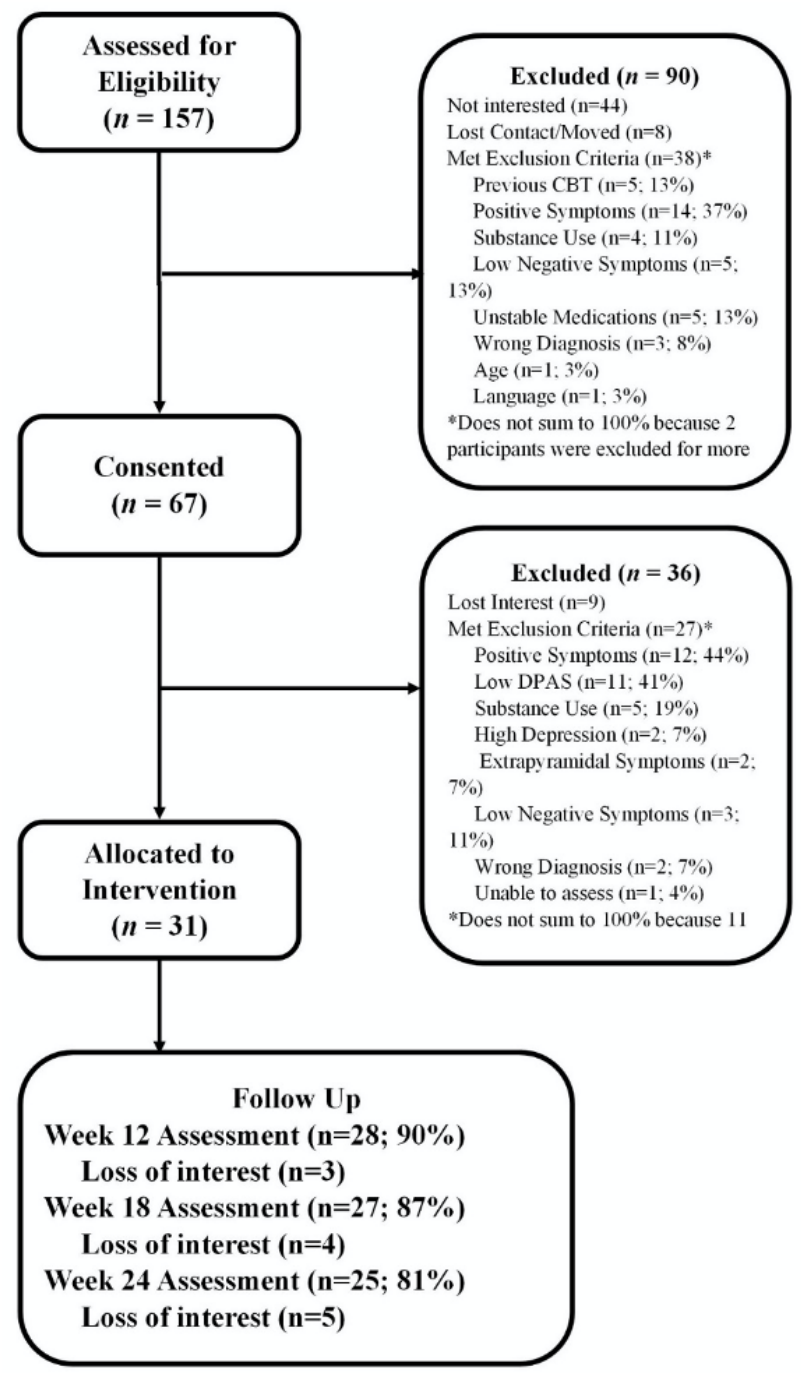


Table 1. Outcome variables and paired $t$ tests at each assessment point relative to baseline.

\begin{tabular}{|c|c|c|c|c|c|}
\hline Outcome measure and time points & $\mathrm{n}(\%)$ & Score, mean (SD) & Cohen $d^{\mathrm{a}}$ & 2-tailed $t$ test $(d f)$ & $P$ value \\
\hline \multicolumn{6}{|c|}{ Defeatist Performance Attitude Scale } \\
\hline Baseline & $31(100)$ & $66.3(14.4)$ & $\mathrm{N} / \mathrm{A}^{\mathrm{b}}$ & N/A & N/A \\
\hline 12 Weeks & $28(90)$ & $60.7(15.9)$ & 0.40 & $2.23(27)$ & .03 \\
\hline 18 Weeks & $27(87)$ & $56.4(15.7)$ & 0.70 & $4.02(26)$ & $<.001$ \\
\hline 24 Weeks & $25(81)$ & $52.2(17.3)$ & 1.00 & $4.10(24)$ & $<.001$ \\
\hline \multicolumn{6}{|l|}{ Asocial Beliefs Scale } \\
\hline Baseline & $30(100)$ & $5.9(3.1)$ & N/A & N/A & N/A \\
\hline 12 Weeks & $28(93)$ & $6.5(2.9)$ & -0.20 & $0.58(27)$ & .57 \\
\hline 18 Weeks & $27(90)$ & $6.2(3.2)$ & -0.10 & $0.21(26)$ & .84 \\
\hline 24 Weeks & $25(83)$ & $6.4(3.3)$ & -0.15 & $0.19(24)$ & .85 \\
\hline \multicolumn{6}{|c|}{ Clinical Assessment Interview for Negative Symptoms Motivation and Pleasure } \\
\hline Baseline & $31(100)$ & $23.1(3.4)$ & N/A & N/A & N/A \\
\hline 12 Weeks & $28(90)$ & $21.3(6.1)$ & 0.55 & $2.07(27)$ & .048 \\
\hline 18 Weeks & $27(87)$ & $20.5(6.2)$ & 0.75 & $2.95(26)$ & .007 \\
\hline 24 Weeks & $25(81)$ & $20.0(6.8)$ & 0.90 & $3.16(24)$ & .004 \\
\hline \multicolumn{6}{|c|}{ Clinical Assessment Interview for Negative Symptoms Expression } \\
\hline Baseline & $31(100)$ & $4.9(3.2)$ & N/A & N/A & N/A \\
\hline 12 Weeks & $28(90)$ & $4.7(3.6)$ & 0.05 & $0.56(27)$ & .58 \\
\hline 18 Weeks & $27(87)$ & $4.3(3.3)$ & 0.20 & $1.18(26)$ & .25 \\
\hline 24 Weeks & $25(81)$ & $4.4(3.3)$ & 0.15 & $1.11(24)$ & .28 \\
\hline \multicolumn{6}{|c|}{ Positive and Negative Syndrome Scale positive symptoms } \\
\hline Baseline & $31(100)$ & $13.4(4.5)$ & N/A & N/A & N/A \\
\hline 12 Weeks & $28(90)$ & $13.0(4.3)$ & 0.10 & $0.75(27)$ & .46 \\
\hline 18 Weeks & $26(84)$ & $12.8(4.8)$ & 0.10 & $0.64(25)$ & .52 \\
\hline 24 Weeks & $24(77)$ & $11.5(4.0)$ & 0.45 & $2.46(23)$ & .02 \\
\hline \multicolumn{6}{|l|}{ Calgary Depression Scale } \\
\hline Baseline & $31(100)$ & $3.4(2.2)$ & N/A & N/A & N/A \\
\hline 12 Weeks & $28(90)$ & $3.4(2.6)$ & 0 & $0(27)$ & $>.99$ \\
\hline 18 Weeks & $27(87)$ & $3.1(2.5)$ & 0.10 & $0.85(26)$ & .40 \\
\hline 24 Weeks & $25(81)$ & $2.7(2.5)$ & 0.30 & $1.99(24)$ & .06 \\
\hline \multicolumn{6}{|l|}{ Abbreviated Quality of Life Scale } \\
\hline Baseline & $31(100)$ & $23.3(6.1)$ & N/A & N/A & N/A \\
\hline 12 Weeks & $28(90)$ & $25.8(7.9)$ & 0.40 & $2.17(27)$ & .04 \\
\hline 18 Weeks & $27(87)$ & $24.9(8.3)$ & 0.25 & $1.78(26)$ & .09 \\
\hline 24 Weeks & $25(81)$ & $24.8(8.8)$ & 0.25 & $1.77(24)$ & .09 \\
\hline \multicolumn{6}{|l|}{ Social Functioning Scale } \\
\hline Baseline & $31(100)$ & $114.7(21.8)$ & N/A & N/A & N/A \\
\hline 12 Weeks & $27(87)$ & $118.5(22.2)$ & 0.15 & $2.27(26)$ & .03 \\
\hline 18 Weeks & $27(87)$ & $115.9(23.6)$ & 0.05 & $1.70(26)$ & .10 \\
\hline 24 Weeks & $25(81)$ & $117.3(26.7)$ & 0.10 & $1.70(24)$ & .10 \\
\hline
\end{tabular}

${ }^{\mathrm{a}} \mathrm{A}$ positive Cohen $d$ value indicates improvement on the outcome from baseline to follow-up. 
${ }^{\mathrm{b}} \mathrm{N} / \mathrm{A}$ : not applicable.

\section{Dysfunctional Attitudes}

The effect of time was significant for defeatist performance attitudes (DPAS: $\left.\gamma=-0.59, t_{30}=-4.27, P<.001\right)$. Change in DPAS score from baseline (see Table 1) was significant at all assessment points during treatment, with medium to large effect sizes. The minimal treatment dose needed to achieve at least a medium effect size was at 18 weeks. In contrast, the effect of time was not significant for asocial beliefs (ABS: $\gamma=0.01$, $\left.t_{29}=0.47, P=.64\right)$.

\section{Symptoms}

Significant reduction in severity of experiential negative symptoms was found (CAINS-MAP: $\gamma=-0.14, t_{30}=-3.12$, $P=.004$ ) with medium to large effect sizes (see Table 1). The minimal treatment dose needed to achieve at least a medium effect size was at 12 weeks. For expressive negative symptoms, assessed using the CAINS Expression (CAINS-EXP) subscale, the effect of time was not statistically significant and no significant reduction from baseline was found at any assessment point (CAINS-EXP: $\gamma=-0.02, t_{30}=-1.06, P=.30$ ). Significant reduction in severity of positive symptoms was found by week 24, but not at earlier assessment points, and the effect of time was not significant (PANSS-positive subscale: $\gamma=-0.06$, $\left.t_{30}=-1.46, P=.16\right)$. Similarly, significant reduction in severity of depressive symptoms was found by week 24 but not at earlier assessment points, and the effect of time was not significant (CDS: $\left.\gamma=-0.03, t_{30}=-1.66, P=.11\right)$.

\section{Mobile Symptom and Attitude Ratings}

CBT2go app ratings of motivation and anticipated pleasure and success for completing planned activities increased significantly for motivation $\left(\gamma=0.007, t_{28}=2.17, P=.04\right)$ and anticipated pleasure $\left(\gamma=0.008, t_{28}=2.53, P=.02\right)$ but not for anticipated success $\left(\gamma=0.006, t_{28}=1.62, P=.12\right)$. This indicates steady gains in self-reported motivation and pleasure (0.7-0.8 points per 100 days on a $0-7$ scale) over treatment. To estimate effect sizes for these changes over the course of treatment, we computed the mean ratings for the first 4 weeks and last 4 weeks of treatment for mobile CBT2go app ratings. Large increases in mean ratings were found between the first 4 weeks of treatment and the last 4 weeks for motivation (Cohen $d=0.65$; first 4 weeks: mean 2.9, SD 1.8; last 4 weeks: mean 4.1, SD 1.8) and anticipated pleasure (Cohen $d=0.80$; first 4 weeks: mean 3.0, SD 1.5; last 4 weeks: mean 4.2, SD 2.1). Medium increases were found for anticipated success (Cohen $d=0.55$; first 4 weeks: mean 3.1, SD 1.5; last 4 weeks: mean 3.9, SD 1.9).

\section{Functioning}

Significant improvement in A-QLS scores was found between baseline and 12 weeks but not at other assessment points, and the effect of time was not significant $\left(\gamma=0.08, t_{30}=1.49, P=.15\right)$. Participants also showed significant improvement on the SFS total score between baseline and 12 weeks but not at the other two assessment points, and the effect of time was only at a trend level $\left(\gamma=0.19, t_{30}=1.77, P=.09\right)$.

\section{App Engagement}

The CBT2go app prompted participants to select an action plan each day during up to 168 days of treatment. There was a mean of 18.7 (SD 21.3) responses to 84 action plan prompts (22\%) at 12 weeks and a mean of 32.3 (SD 31.5) responses to 168 action plan prompts $(19.2 \%)$ at 24 weeks for participants who did not drop out of treatment by each assessment point; this indicates engagement in homework and skills practice more than once per week, with minimal fatigue effects over the course of treatment. The number of action plans completed was not significantly correlated with any symptom measure at baseline (range of $r=-0.05$ to 0.07 ).

In HLM analyses examining the association between app engagement and outcome, the number of action plans by time interaction was significant for change in CAINS-MAP scores $\left(\gamma=-0.003, t_{29}=-2.11, P=.04\right)$ but not for DPAS scores $\left(\gamma=-0.002, t_{29}=-0.36, P=.72\right)$. The change in CAINS-MAP scores relative to baseline was also marginally correlated with the number of action plans completed at 12 weeks $(r=-0.33$, $P=.09)$ and 18 weeks $(r=-0.35, P=.07)$ but not 24 weeks $(r=-0.28, P=18)$. This did not hold true for change in DPAS scores relative to baseline at 12 weeks $(r=-0.23, P=.24), 18$ weeks $(r=-0.13, P=.51)$, and 24 weeks $(r=-0.07, P=.75)$. Thus, greater engagement with the app was associated with greater reduction in motivation and pleasure negative symptoms.

\section{Discussion}

The results of this open trial of mCBTn showed significant, large, within-group improvements in defeatist attitudes and negative symptoms and defeatist attitudes by 18 weeks of treatment, which demonstrates feasibility and engagement of the defeatist attitudes target, and justifies a larger RCT. These findings also provide support for the defeatist attitude model of negative symptoms $[10,12]$. Some specificity was found for improvements in defeatist performance beliefs but not asocial beliefs, perhaps because the intervention focused on all living, learning, working, and socializing goals, rather than only socialization. The intensive focus of mCBTn on modifying defeatist beliefs, both in group therapy and in real-world environments using the CBT2go app, resulted in a large improvement in experiential negative symptoms within a relatively short 18-week treatment duration and medium improvement by 12 weeks, which is relatively rapid change. If blended interventions such as mCBTn can strengthen and thereby shorten intensive psychosocial interventions, then implementation barriers associated with burden and cost of lengthy psychotherapy interventions would be reduced. This would improve access to evidence-based practices for consumers with severe and persistent negative symptoms, which would have considerable public health significance. However, further testing in a larger RCT with a control condition is needed to confirm the efficacy of mCBTn and justify work on implementation.

This study adds to the growing literature on CBT-based mobile interventions for schizophrenia $[37,38,48,49]$ and indicates that 
patients with severe and persistent negative symptoms may be amenable to this approach. In the first trial to use mobile CBT-informed interventions for schizophrenia in a text-messaging platform, we [35] used a similar approach that integrated EMA of symptoms and behaviors (eg, social isolation, medication adherence, and voices) in everyday contexts and delivered personalized just-in-time thought-challenging messages when participants reported symptom distress or defeatist attitudes; we also found improvements in auditory hallucinations, socialization, and medication adherence. In another prior trial using this same algorithm in an earlier version of the CBT2go app, we found improvements in total symptoms in a large RCT of patients with schizophrenia and bipolar disorder [36]. Ben-Zeev and colleagues [50,51] also used a similar CBT-informed algorithm with the FOCUS app and added sleep interventions and on-demand educational components; they found reductions in several symptom domains in schizophrenia. Finally, Bucci et al [52] developed the Actissist app, which uses an algorithm based on the cognitive model of psychosis, and found large improvements in symptoms relative to symptom monitoring only in participants with early psychosis.

Related to this, recent meta-analyses have suggested that SST may be a more effective treatment for negative symptoms of schizophrenia than CBT $[28,29]$. The mCBTn intervention in this study did not include the SST or problem-solving components included in CBSST. Thus, while SST may be an effective treatment for negative symptoms, this trial suggests that the CBT components of CBSST targeting defeatist attitudes can improve experiential negative symptoms without SST.

With regard to secondary outcomes, modest improvements were found in positive symptoms and depression with a longer 24-week treatment period, which was not expected, given that participants were screened for severe positive symptoms and depression. Changes in functioning were mixed, with significant improvements found early in treatment but then dissipated with only trend-level improvements found overall on the A-QLS and SFS. The 24-week follow-up period may be too brief to expect meaningful changes in functioning.

This study had a high exclusion rate during the run-in period, with 36 out of 67 (54\%) participants not meeting the strict, persistent, negative symptom entry criteria. A high screen failure rate during run-in periods is common in clinical trials with similar persistent negative symptom criteria. For example, a screen failure rate of $44 \%$ was found in a psychosocial trial using similar criteria, except DPAS [26], and this rate is slightly higher than in pharmaceutical trials with similar criteria [26,53]. The proportion of patients with documented persistent negative symptoms (46\%) is consistent with other estimates [3] and suggests negative symptoms are a common treatment need in patients with schizophrenia. Clinical trials of participants with persistent negative symptoms are rare, so this study makes an important contribution by demonstrating improvements in experiential negative symptoms as a primary target rather than secondary improvements related to changes in positive or depressive symptoms. It is important to note that participants were also recruited specifically for persistent experiential negative symptoms, which are more strongly linked to functional impairments than expressive symptoms $[5,7,54,55]$.

Retention rates were excellent (81\%-90\% across assessments), especially for this negative symptom population, suggesting the intervention is feasible. It may be important that transportation was provided to therapy groups, which likely facilitated retention and may be necessary to maintain engagement of this population, especially in a large county with limited public transportation where this study was conducted. We have found much better retention in CBSST trials when transportation was provided [56] than when it was not [57]. The CBT2go app may have also promoted engagement, for example, through daily reminders of personalized recovery goals. The mCBTn intervention focused on recovery goal work, which can improve motivation and promote engagement in psychiatric rehabilitation [58].

Engagement with the CBT2go app was mixed. The app was designed to promote engagement in recovery activities as often as every day. On average, however, participants responded to prompts to make an action plan for the day about one and a half times per week. While this proportion of days with completed action plans may seem low, practicing skills and completing homework assignments more than once per week is greater homework adherence than would be expected with CBT group therapy alone, where participants are typically expected to complete a single homework assignment per week. Homework adherence in CBT psychosocial interventions across multiple disorders is approximately 20\%-56\%. Thus, completing approximately one and a half action plans per week is better community engagement in recovery activities than might be expected in CBT therapy alone with one assignment per week. Greater engagement with the app was also associated with greater improvement in motivational negative symptoms and was unrelated to baseline severity of negative symptoms, suggesting the app played an important role in strengthening the treatment's impact on this important outcome.

This trial had several limitations. First, as described above, further app development is needed to promote engagement (eg, simplified interface and rewards and feedback to motivate). In addition, patients with greater severity of defeatist attitudes were recruited, because this was the target mechanism, so the findings may not generalize to patients whose experiential negative symptoms may not be related to defeatist attitudes. Participants were also excluded for severe positive symptoms or depression, so findings may not generalize to these populations. Finally, and importantly, this was a preliminary open trial that did not control for the effects of time, therapist contact, trips out of the home to come to group, socialization with staff and other patients, and other nonspecific factors. The next step is to complete an RCT with a contact control condition, which we are currently conducting. If this ongoing RCT with the modified CBT2go app confirms the findings of this open trial, this would provide stronger support for the defeatist attitude model of experiential negative symptoms and suggest that blended interventions like mCBTn can strengthen and shorten intensive psychosocial interventions for negative symptoms in schizophrenia. 


\section{Acknowledgments}

We thank the participants who volunteered for this study. Research reported in this publication was supported by the National Institute of Mental Health (principal investigator EG; R61MH110019). This open trial was funded as part of the National Institute of Mental Health Experimental Therapeutics Program (RFA-MH-18-704 R61/R33). The content is solely the responsibility of the authors and does not necessarily represent the official views of the Department of Veterans Affairs or National Institutes of Health.

\section{Conflicts of Interest}

EG has an equity interest in Granholm Consulting, Inc, and may benefit from the research results as he receives income from the company for CBSST workshops and consulting. The terms of this arrangement have been reviewed and approved by the University of California, San Diego, in accordance with its conflict of interest policies. All other authors have no conflicts of interest to declare.

\section{References}

1. Fleischhacker WW, Arango C, Arteel P, Barnes TRE, Carpenter W, Duckworth K, et al. Schizophrenia--Time to commit to policy change. Schizophr Bull 2014 Apr;40 Suppl 3:S165-S194 [FREE Full text] [doi: 10.1093/schbul/sbu006] [Medline: 24778411]

2. Kirkpatrick B, Fenton WS, Carpenter WT, Marder SR. The NIMH-MATRICS consensus statement on negative symptoms. Schizophr Bull 2006 Apr;32(2):214-219 [FREE Full text] [doi: 10.1093/schbul/sbj053] [Medline: 16481659]

3. Buchanan RW. Persistent negative symptoms in schizophrenia: An overview. Schizophr Bull 2007 Jul;33(4):1013-1022 [FREE Full text] [doi: 10.1093/schbul/sbl057] [Medline: 17099070]

4. Blanchard JJ, Cohen AS. The structure of negative symptoms within schizophrenia: Implications for assessment. Schizophr Bull 2006 Apr;32(2):238-245 [FREE Full text] [doi: 10.1093/schbul/sbj013] [Medline: 16254064]

5. Kring AM, Gur RE, Blanchard JJ, Horan WP, Reise SP. The Clinical Assessment Interview for Negative Symptoms (CAINS): Final development and validation. Am J Psychiatry 2013 Feb;170(2):165-172 [FREE Full text] [doi: 10.1176/appi.ajp.2012.12010109] [Medline: 23377637]

6. Marder SR, Kirkpatrick B. Defining and measuring negative symptoms of schizophrenia in clinical trials. Eur Neuropsychopharmacol 2014 May;24(5):737-743. [doi: 10.1016/j.euroneuro.2013.10.016] [Medline: 24275698]

7. Rassovsky Y, Horan WP, Lee J, Sergi MJ, Green MF. Pathways between early visual processing and functional outcome in schizophrenia. Psychol Med 2010 May 19;41(3):487-497. [doi: 10.1017/s0033291710001054]

8. Buchanan RW, Kreyenbuhl J, Kelly DL, Noel JM, Boggs DL, Fischer BA, Schizophrenia Patient Outcomes Research Team (PORT). The 2009 schizophrenia PORT psychopharmacological treatment recommendations and summary statements. Schizophr Bull 2010 Jan;36(1):71-93 [FREE Full text] [doi: 10.1093/schbul/sbp116] [Medline: 19955390]

9. Dixon LB, Dickerson F, Bellack AS, Bennett M, Dickinson D, Goldberg RW, Schizophrenia Patient Outcomes Research Team (PORT). The 2009 schizophrenia PORT psychosocial treatment recommendations and summary statements. Schizophr Bull 2010 Jan;36(1):48-70 [FREE Full text] [doi: 10.1093/schbul/sbp115] [Medline: 19955389]

10. Grant PM, Beck AT. Defeatist beliefs as a mediator of cognitive impairment, negative symptoms, and functioning in schizophrenia. Schizophr Bull 2009 Jul;35(4):798-806 [FREE Full text] [doi: 10.1093/schbul/sbn008] [Medline: 18308717]

11. Rector NA, Beck AT, Stolar N. The negative symptoms of schizophrenia: A cognitive perspective. Can J Psychiatry 2005 Apr;50(5):247-257. [doi: 10.1177/070674370505000503] [Medline: 15968839]

12. Beck A, Rector N, Stolar N, Grant P. Schizophrenia: Cognitive Theory, Research, and Therapy. New York, NY: The Guilford Press; 2009.

13. Campellone TR, Sanchez AH, Kring AM. Defeatist performance beliefs, negative symptoms, and functional outcome in schizophrenia: A meta-analytic review. Schizophr Bull 2016 Nov;42(6):1343-1352 [FREE Full text] [doi: 10.1093/schbul/sbw026] [Medline: 26980144]

14. Couture SM, Blanchard JJ, Bennett ME. Negative expectancy appraisals and defeatist performance beliefs and negative symptoms of schizophrenia. Psychiatry Res 2011 Aug 30;189(1):43-48 [FREE Full text] [doi: 10.1016/j.psychres.2011.05.032] [Medline: 21704387]

15. Granholm E, Ben-Zeev D, Link PC. Social disinterest attitudes and group cognitive-behavioral social skills training for functional disability in schizophrenia. Schizophr Bull 2009 Sep;35(5):874-883 [FREE Full text] [doi: 10.1093/schbul/sbp072] [Medline: 19628761$]$

16. Horan WP, Rassovsky Y, Kern RS, Lee J, Wynn JK, Green MF. Further support for the role of dysfunctional attitudes in models of real-world functioning in schizophrenia. J Psychiatr Res 2010 Jun;44(8):499-505 [FREE Full text] [doi:

10.1016/j.jpsychires.2009.11.001] [Medline: 20006849]

17. Quinlan T, Roesch S, Granholm E. The role of dysfunctional attitudes in models of negative symptoms and functioning in schizophrenia. Schizophr Res 2014 Aug;157(1-3):182-189 [FREE Full text] [doi: 10.1016/j.schres.2014.05.025] [Medline: 24924405] 
18. Green MF, Hellemann G, Horan WP, Lee J, Wynn JK. From perception to functional outcome in schizophrenia: Modeling the role of ability and motivation. Arch Gen Psychiatry 2012 Dec;69(12):1216-1224 [FREE Full text] [doi: 10.1001/archgenpsychiatry.2012.652] [Medline: 23026889]

19. Bandura A, Freeman WH, Lightsey R. Self-efficacy: The exercise of control. J Cogn Psychother 1999 Jan 01;13(2):158-166. [doi: 10.1891/0889-8391.13.2.158]

20. Velthorst E, Koeter M, van der Gaag M, Nieman DH, Fett AJ, Smit F, et al. Adapted cognitive-behavioural therapy required for targeting negative symptoms in schizophrenia: Meta-analysis and meta-regression. Psychol Med 2015 Feb;45(3):453-465. [doi: 10.1017/S0033291714001147] [Medline: 24993642]

21. Jauhar S, McKenna PJ, Radua J, Fung E, Salvador R, Laws KR. Cognitive-behavioural therapy for the symptoms of schizophrenia: Systematic review and meta-analysis with examination of potential bias. Br J Psychiatry 2014 Jan;204(1):20-29. [doi: 10.1192/bjp.bp.112.116285] [Medline: 24385461]

22. Grant PM, Huh GA, Perivoliotis D, Stolar NM, Beck AT. Randomized trial to evaluate the efficacy of cognitive therapy for low-functioning patients with schizophrenia. Arch Gen Psychiatry 2012 Feb;69(2):121-127. [doi: 10.1001/archgenpsychiatry.2011.129] [Medline: 21969420]

23. Granholm E, Holden J, Worley M. Improvement in negative symptoms and functioning in cognitive-behavioral social skills training for schizophrenia: Mediation by defeatist performance attitudes and asocial beliefs. Schizophr Bull 2018 Apr 06;44(3):653-661 [FREE Full text] [doi: 10.1093/schbul/sbx099] [Medline: 29036391]

24. Perivoliotis D, Cather C. Cognitive behavioral therapy of negative symptoms. J Clin Psychol 2009 Aug;65(8):815-830. [doi: 10.1002/jclp.20614] [Medline: 19572278]

25. Staring ABP, Ter Huurne MB, van der Gaag M. Cognitive Behavioral Therapy for negative symptoms (CBT-n) in psychotic disorders: A pilot study. J Behav Ther Exp Psychiatry 2013 Sep;44(3):300-306. [doi: 10.1016/j.jbtep.2013.01.004] [Medline: 23454550]

26. Velligan D, Roberts D, Mintz J, Maples N, Li X, Medellin E, et al. A randomized pilot study of MOtiVation and Enhancement (MOVE) Training for negative symptoms in schizophrenia. Schizophr Res 2015 Jul;165(2-3):175-180 [FREE Full text] [doi: 10.1016/j.schres.2015.04.008] [Medline: 25937461]

27. Morrison AP, Turkington D, Wardle M, Spencer H, Barratt S, Dudley R, et al. A preliminary exploration of predictors of outcome and cognitive mechanisms of change in cognitive behaviour therapy for psychosis in people not taking antipsychotic medication. Behav Res Ther 2012 Feb;50(2):163-167. [doi: 10.1016/j.brat.2011.12.001] [Medline: 22209267]

28. Kurtz MM, Mueser KT. A meta-analysis of controlled research on social skills training for schizophrenia. J Consult Clin Psychol 2008 Jun;76(3):491-504. [doi: 10.1037/0022-006X.76.3.491] [Medline: 18540742]

29. Turner DT, McGlanaghy E, Cuijpers P, van der Gaag M, Karyotaki E, MacBeth A. A meta-analysis of social skills training and related interventions for psychosis. Schizophr Bull 2018 Apr 06;44(3):475-491 [FREE Full text] [doi: 10.1093/schbul/sbx146] [Medline: 29140460]

30. Granholm EL, McQuaid JR, Holden JL. Cognitive-Behavioral Social Skills Training for Schizophrenia: A Practical Treatment Guide. New York, NY: The Guilford Press; 2016.

31. Ben-Zeev D, Davis KE, Kaiser S, Krzsos I, Drake RE. Mobile technologies among people with serious mental illness: Opportunities for future services. Adm Policy Ment Health 2013 Jul;40(4):340-343 [FREE Full text] [doi: 10.1007/s10488-012-0424-x] [Medline: 22648635]

32. Brunette M, Achtyes E, Pratt S, Stilwell K, Opperman M, Guarino S, et al. Use of smartphones, computers and social media among people with SMI: Opportunity for intervention. Community Ment Health J 2019 Aug;55(6):973-978. [doi: 10.1007/s10597-019-00431-7] [Medline: 31175518]

33. Lal S, Dell'Elce J, Malla AK. Technology access and use among young adults with a first episode of psychosis. Psychiatr Serv 2015 Jul;66(7):764-765. [doi: 10.1176/appi.ps.201400580] [Medline: 26130154]

34. Firth J, Cotter J, Torous J, Bucci S, Firth JA, Yung AR. Mobile phone ownership and endorsement of "mHealth" among people with psychosis: A meta-analysis of cross-sectional studies. Schizophr Bull 2016 Mar;42(2):448-455 [FREE Full text] [doi: 10.1093/schbul/sbv132] [Medline: 26400871]

35. Granholm E, Ben-Zeev D, Link PC, Bradshaw KR, Holden JL. Mobile Assessment and Treatment for Schizophrenia (MATS): A pilot trial of an interactive text-messaging intervention for medication adherence, socialization, and auditory hallucinations. Schizophr Bull 2012 May;38(3):414-425 [FREE Full text] [doi: 10.1093/schbul/sbr155] [Medline: 22080492]

36. Depp CA, Perivoliotis D, Holden J, Dorr J, Granholm EL. Single-session mobile-augmented intervention in serious mental illness: A three-arm randomized controlled trial. Schizophr Bull 2019 Jun 18;45(4):752-762 [FREE Full text] [doi: 10.1093/schbul/sby135] [Medline: 30281086]

37. Camacho E, Levin L, Torous J. Smartphone apps to support coordinated specialty care for prodromal and early course schizophrenia disorders: Systematic review. J Med Internet Res 2019 Nov 12;21(11):e16393 [FREE Full text] [doi: 10.2196/16393] [Medline: 31714250]

38. Firth J, Torous J. Smartphone apps for schizophrenia: A systematic review. JMIR Mhealth Uhealth 2015 Nov 06;3(4):e102 [FREE Full text] [doi: 10.2196/mhealth.4930] [Medline: 26546039]

39. Bell I, Rossell S, Farhall J, Hayward M, Lim M, Fielding-Smith S, et al. Pilot randomised controlled trial of a brief coping-focused intervention for hearing voices blended with smartphone-based ecological momentary assessment and 
intervention (SAVVy): Feasibility, acceptability and preliminary clinical outcomes. Schizophr Res 2020 Feb;216:479-487. [doi: 10.1016/j.schres.2019.10.026] [Medline: 31812327]

40. Schlosser D, Campellone T, Kim D, Truong B, Vergani S, Ward C, et al. Feasibility of PRIME: A cognitive neuroscience-informed mobile app intervention to enhance motivated behavior and improve quality of life in recent onset schizophrenia. JMIR Res Protoc 2016 Apr 28;5(2):e77 [FREE Full text] [doi: 10.2196/resprot.5450] [Medline: 27125771]

41. Schlosser DA, Campellone TR, Truong B, Etter K, Vergani S, Komaiko K, et al. Efficacy of PRIME, a mobile app intervention designed to improve motivation in young people with schizophrenia. Schizophr Bull 2018 Aug 20;44(5):1010-1020 [FREE Full text] [doi: 10.1093/schbul/sby078] [Medline: 29939367]

42. Kay SR, Fiszbein A, Opler LA. The positive and negative syndrome scale (PANSS) for schizophrenia. Schizophr Bull 1987;13(2):261-276. [doi: 10.1093/schbul/13.2.261] [Medline: 3616518]

43. Addington D, Addington J, Schissel B. A depression rating scale for schizophrenics. Schizophr Res 1990;3(4):247-251. [doi: 10.1016/0920-9964(90)90005-r] [Medline: 2278986]

44. Simpson GM, Angus JW. A rating scale for extrapyramidal side effects. Acta Psychiatr Scand Suppl 1970;212:11-19. [doi: 10.1111/j.1600-0447.1970.tb02066.x] [Medline: 4917967]

45. Grant PM, Beck AT. Asocial beliefs as predictors of asocial behavior in schizophrenia. Psychiatry Res 2010 May 15;177(1-2):65-70. [doi: 10.1016/j.psychres.2010.01.005] [Medline: 20163875]

46. Fervaha G, Foussias G, Siddiqui I, Agid O, Remington G. Abbreviated quality of life scales for schizophrenia: Comparison and utility of two brief community functioning measures. Schizophr Res 2014 Apr;154(1-3):89-92. [doi: 10.1016/j.schres.2014.02.013] [Medline: 24630140]

47. Birchwood M, Smith J, Cochrane R, Wetton S, Copestake S. The Social Functioning Scale. The development and validation of a new scale of social adjustment for use in family intervention programmes with schizophrenic patients. Br J Psychiatry 1990 Dec;157:853-859. [doi: 10.1192/bjp.157.6.853] [Medline: 2289094]

48. Bell IH, Lim MH, Rossell SL, Thomas N. Ecological momentary assessment and intervention in the treatment of psychotic disorders: A systematic review. Psychiatr Serv 2017 Nov 01;68(11):1172-1181. [doi: 10.1176/appi.ps.201600523] [Medline: 28669284]

49. Alvarez-Jimenez M, Alcazar-Corcoles MA, González-Blanch C, Bendall S, McGorry PD, Gleeson JF. Online, social media and mobile technologies for psychosis treatment: A systematic review on novel user-led interventions. Schizophr Res 2014 Jun;156(1):96-106. [doi: 10.1016/j.schres.2014.03.021] [Medline: 24746468]

50. Ben-Zeev D, Brenner CJ, Begale M, Duffecy J, Mohr DC, Mueser KT. Feasibility, acceptability, and preliminary efficacy of a smartphone intervention for schizophrenia. Schizophr Bull 2014 Nov;40(6):1244-1253 [FREE Full text] [doi: 10.1093/schbul/sbu033] [Medline: 24609454]

51. Ben-Zeev D, Kaiser SM, Brenner CJ, Begale M, Duffecy J, Mohr DC. Development and usability testing of FOCUS: A smartphone system for self-management of schizophrenia. Psychiatr Rehabil J 2013 Dec;36(4):289-296 [FREE Full text] [doi: 10.1037/prj0000019] [Medline: 24015913]

52. Bucci S, Barrowclough C, Ainsworth J, Machin M, Morris R, Berry K, et al. Actissist: Proof-of-concept trial of a theory-driven digital intervention for psychosis. Schizophr Bull 2018 Aug 20;44(5):1070-1080 [FREE Full text] [doi: 10.1093/schbul/sby032] [Medline: 29566206]

53. Rabinowitz J, Werbeloff N, Caers I, Mandel FS, Stauffer V, Menard F, et al. Negative symptoms in schizophrenia--The remarkable impact of inclusion definitions in clinical trials and their consequences. Schizophr Res 2013 Nov;150(2-3):334-338. [doi: 10.1016/j.schres.2013.06.023] [Medline: 23815975]

54. Blanchard JJ, Bradshaw KR, Garcia CP, Nasrallah HA, Harvey PD, Casey D, et al. Examining the reliability and validity of the Clinical Assessment Interview for Negative Symptoms within the Management of Schizophrenia in Clinical Practice (MOSAIC) multisite national study. Schizophr Res 2017 Jul;185:137-143. [doi: 10.1016/j.schres.2017.01.011] [Medline: $\underline{28087270]}$

55. Rocca P, Montemagni C, Zappia S, Piterà R, Sigaudo M, Bogetto F. Negative symptoms and everyday functioning in schizophrenia: A cross-sectional study in a real world-setting. Psychiatry Res 2014 Aug 30;218(3):284-289. [doi: 10.1016/j.psychres.2014.04.018] [Medline: 24814140]

56. Granholm E, Holden J, Link PC, McQuaid JR, Jeste DV. Randomized controlled trial of cognitive behavioral social skills training for older consumers with schizophrenia: Defeatist performance attitudes and functional outcome. Am J Geriatr Psychiatry 2013 Mar;21(3):251-262 [FREE Full text] [doi: 10.1016/j.jagp.2012.10.014] [Medline: 23395192]

57. Granholm E, Holden J, Link PC, McQuaid JR. Randomized clinical trial of cognitive behavioral social skills training for schizophrenia: Improvement in functioning and experiential negative symptoms. J Consult Clin Psychol 2014 Dec;82(6):1173-1185 [FREE Full text] [doi: 10.1037/a0037098] [Medline: 24911420]

58. Clarke SP, Oades LG, Crowe TP, Deane FP. Collaborative goal technology: Theory and practice. Psychiatr Rehabil J 2006;30(2):129-136. [doi: 10.2975/30.2006.129.136] [Medline: 17076056 ]

\section{Abbreviations}

ABS: Asocial Beliefs Scale 
A-QLS: Abbreviated Quality of Life Scale

CAINS-MAP: Clinical Assessment Interview for Negative Symptoms Motivation and Pleasure

CBSST: cognitive behavioral social skills training

CBT: cognitive behavioral therapy

CDS: Calgary Depression Scale for Schizophrenia

CONSORT: Consolidated Standards of Reporting Trials

DPAS: Defeatist Performance Attitude Scale

DSM-5: Diagnostic and Statistical Manual of Mental Disorders, fifth edition

EMA: ecological momentary assessment

HLM: hierarchical linear modeling

MATS: Mobile Assessment and Treatment of Schizophrenia

mCBTn: mobile-assisted cognitive behavioral therapy for negative symptoms

PANSS: Positive and Negative Syndrome Scale

PRIME: Personalized Real-time Intervention for Motivational Enhancement

RCT: randomized controlled trial

SAVVy: Smartphone-Assisted coping-focused interVention for Voices

SCID-5: Structured Clinical Interview for DSM-5

SFS: Social Functioning Scale

SST: social skills training

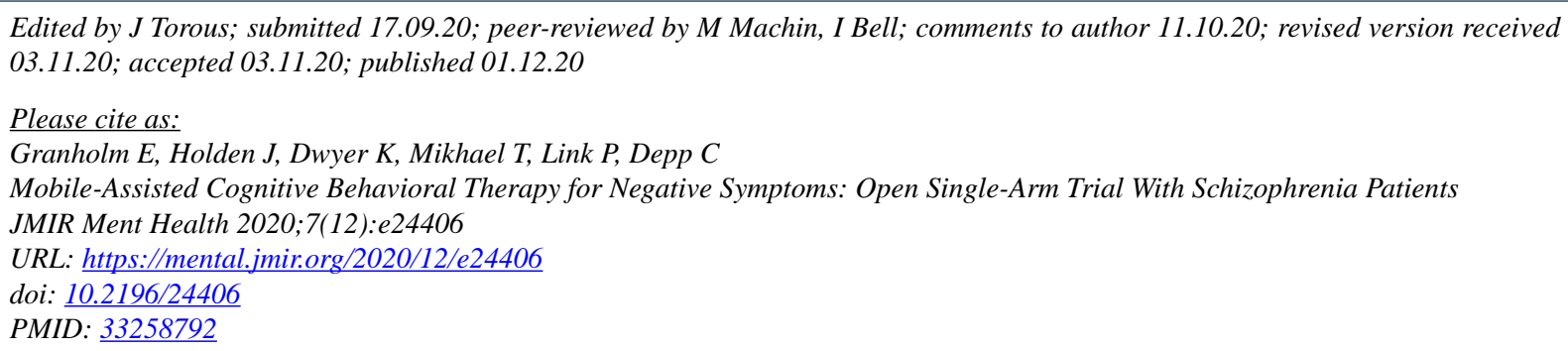

CEric Granholm, Jason Holden, Kristen Dwyer, Tanya Mikhael, Peter Link, Colin Depp. Originally published in JMIR Mental Health (http://mental.jmir.org), 01.12.2020. This is an open-access article distributed under the terms of the Creative Commons Attribution License (https://creativecommons.org/licenses/by/4.0/), which permits unrestricted use, distribution, and reproduction in any medium, provided the original work, first published in JMIR Mental Health, is properly cited. The complete bibliographic information, a link to the original publication on http://mental.jmir.org/, as well as this copyright and license information must be included. 